\title{
Research highlight: inhibition of CSF-IR signaling stimulates PMN-MDSC infiltration and tumor progression
}

\author{
Chinmay R Surve \\ Department of Anatomy and Structural Biology, Albert Einstein college of Medicine, USA
}

Correspondence: Chinmay R Surve PhD, Department of Anatomy and Structural Biology, Albert Einstein college of Medicine, Bronx, NY, USA, Email chinmay.surve@einstein.yu.edu

Received: March 22, 2018 | Published: March 23, 2018

Copyright@ 2018 Surve. This is an open access article distributed under the terms of the Creative Commons Attribution License, which permits unrestricted use, distribution, and reproduction in any medium, provided the original author and source are credited.

\begin{abstract}
CSF-1R signaling pathway is a widely targeted pathway for treatment of cancer patients. The effects of CSF-1R inhibition have been widely attributed to its prevention of macrophage recruitment to the tumors. Last decade has seen the rise in use of CSF-1R inhibitors in clinical trials as a combination with neoadjuvant chemotherapy and immune checkpoint inhibitors. CSF-1R inhibitors though useful were rarely effective in complete cancer cure and disease free survival. This paradox is answered by new findings from and Kuman V and collaborators ${ }^{1}$ who show that though CSF1-R reduces macrophage infiltration, they also recruit polymorphonuclear myeloid-derived suppressor cells, suppressing T-cell function and promoting tumor survival and metastasis.
\end{abstract}

Keywords: cancer, CSF-1R, CSF1, CAFs, tumor progression, Cxc11

\section{Introduction}

CSF-1R inhibitors have been widely used in the last decade for cancer treatment. Recent published work by Kuman V and collaborators show that though CSF1-R reduces macrophage infiltration, they also recruit polymorphonuclear myeloid-derived suppressor cells (PMNMDSC), suppressing T-cell function and promoting tumor survival and metastasis.

Kumar V, et al. ${ }^{1}$ using various mice models of cancer demonstrate that CSF-1R inhibition using a selective inhibitor JNJ-40346527 led to no delay in tumor progression in mice models of melanoma, colon carcinoma, lung carcinoma and lymphoma but led to significant reduction in tumor progression in breast carcinoma. The mechanism which they believe lead to no decrease in tumor progression was through the increase in the number of immuno-suppressive granulocytic cells in all tumor types. The upregulation of granulocyte recruiting chemokines, specifically Cxcl1, a major player in neutrophil and PMN-MDSC recruitment led to increased recruitment of immunosuppressive granulocytic cells to tumors. Next, they demonstrate that cancer associated fibroblasts (CAFs), and not by myeloid or cancer cells secreted Cxcll in tumors. This was confirmed by removing CAFs from tumors using injection of FAP-specific CAR-T cells. The removal of CAFs led to reduced tumor growth, Cxcll expression and PMN-MDSC in tumors. Next, they isolated CAFs from lung carcinoma mouse model and found that CAFs express CSF-1R and secrete Cxcl1. The secretion of Cxcll by CAFs and migration of PMN-MDSC was reduced upon CSF1 stimulation, with inhibition of CSF-1R in CSF1 stimulated CAFs able to restore both back to basal levels. Using knockout of CSF1 in cancer cells, CXCR2 in bone marrow derived cells and CSF1R in CAFs they demonstrated that inhibition CSF-1R signaling eliminated the downregulation of Cxcl1 and accumulation of PMN-MDSC in tumors. Last, the authors used tumors patient samples to further correlate CSF1's role in increased accumulation of PMN-MDSC and in increasing tumor progression. Finally, the authors show that CSF1 regulates Cxcl1 expression in CAFs by recruiting histone deacetylase2 (HDAC2) to the cxcl1 promoter preventing Cxcll expression, secretion, PMN-MDSC recruitment and tumor progression.

\section{References}

1. Kumar V, Donthireddy L, Marvel D, et al. Cancer-Associated Fibroblasts Neutralize the Anti-tumor Effect of CSF1 Receptor Blockade by Inducing PMN-MDSC Infiltration of Tumors. Cancer Cell. 2017;32(5): 654-668. 